\title{
3D imaging of PSD-95 in the mouse brain using the advanced CUBIC method
}

\author{
Huazheng Liang ${ }^{1,2,3^{*}} \mathbb{D}$, Hongqin Wang ${ }^{4}$, Shaoshi Wang ${ }^{1}$, Richard Francis ${ }^{5}$, George Paxinos $^{2}$ and Xufeng Huang ${ }^{4^{*}}$
}

\begin{abstract}
Aims: Postsynaptic density - 95 kDa protein (PSD95) is an important molecule on the postsynaptic membrane. It interacts with many other proteins and plays a pivotal role in learning and memory formation. Its distribution in the brain has been studied previously using in situ hybridization as well as immunohistochemistry. However, these studies are based on 2 dimensional (2D) sections and results are presented with a few sections. The present study aims to show PSD-95 distribution in 3 dimensions (3D) without slicing the brain tissue of C57BL/6 mice into sections using the advanced CUBIC technique.

Methods: Immunofluorescent staining using a PSD-95 antibody was performed on a half of the mouse brain after clarifying it using the advanced CUBIC protocol. The brain tissue was imaged using a Zeiss Z1 light sheet microscope and 3D reconstruction was completed using the Arivis Vision 4 dimensional (4D) software.

Results: The majority of brain nuclei have similar distribution pattern to what has been reported from in situ hybridization and immunohistochemical studies in the mouse. The signal can be easily followed in the 3D and their spatial relationship with adjacent structures clearly demarcated. In the present study, some fiber bundles also showed strong PSD-95 signal, which is different from what was shown in previous studies and need to be confirmed in future studies.
\end{abstract}

Keywords: PSD-95, Mouse, Advanced CUBIC, Transparent brain, Immunofluorescent staining

\section{Main text}

Postsynaptic density protein of $95 \mathrm{kDa}$ (PSD-95) is a scaffolding protein encoded by the disc large homolog 4 (DLG4) gene. It is highly enriched in the postsynaptic membrane and interacts with multiple synaptic proteins [1] through its three Post synaptic density protein, Drosophila disc large tumor suppressor, and Zonula occludens-1 protein (PDZ) domains. It plays an important role in synaptic plasticity [2], learning and memory [3], and other functions depending on the proteins it binds to. The expression of PSD-95 in the mouse brain has been described in traditional studies [4-6]. PSD-95 is highly expressed in the cerebral cortex, hippocampus, and striatum, and only moderately or weakly expressed in other brain regions. Our research has focused on the

\footnotetext{
* Correspondence: a.liang@westernsydney.edu.au; xhuang@uow.edu.au ${ }^{1}$ Neurology Department, Shanghai No.1 People's Hospital (in the process of affiliation), School of Medicine, Tongji University, Shanghai 200081, China ${ }^{4}$ Illawarra Health and Medical Research Institute, Wollongong, NSW 2522, Australia

Full list of author information is available at the end of the article
}

molecular and histological changes in the brain of disrupted in schizophrenia 1-locus impairment (DISC1-LI) mice, which is known to be relevant to the neuropathology of schizophrenia. The 3D imaging results presented here were from the C57BL/6 mouse as part of our histological analysis comparing the expression changes of PSD-95 in DISC1-LI mice with that of control C57BL/6 mice in the 3D space.

Brains of 12-week old C57BL/6 male mice were fixed with $4 \%$ paraformaldehyde and dissected before being cut into two halves sagittally. Immunofluorescent staining using a PSD-95 antibody was performed on a half of the brains after clarifying them using the advanced CUBIC protocol [7]. The brain tissue was imaged with a Zeiss Z1 light sheet microscope using a $20 \times$ clearing objective and 3D reconstruction was completed using the Arivis Vision 4 dimensional (4D) software.

In the forebrain, we found strong positive PSD-95 signal in the internal plexiform and the mitral cell layers of the olfactory bulb (Fig. 1a and b), which was similar to what

(c) The Author(s). 2018 Open Access This article is distributed under the terms of the Creative Commons Attribution 4.0 International License (http://creativecommons.org/licenses/by/4.0/), which permits unrestricted use, distribution, and reproduction in any medium, provided you give appropriate credit to the original author(s) and the source, provide a link to the Creative Commons license, and indicate if changes were made. The Creative Commons Public Domain Dedication waiver (http://creativecommons.org/publicdomain/zero/1.0/) applies to the data made available in this article, unless otherwise stated. 

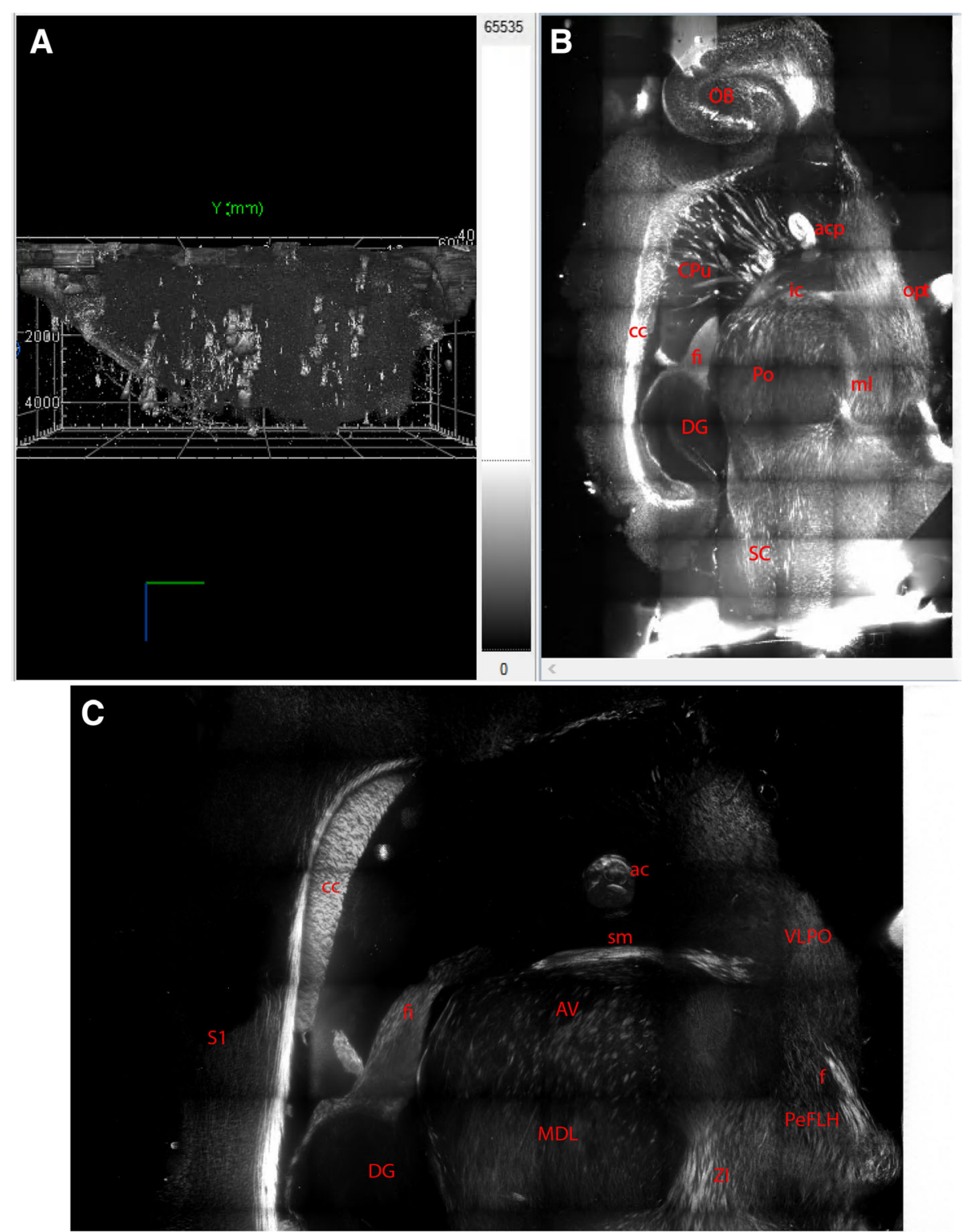

Fig. 1 Fluorescent staining with a PSD-95 antibody on the clarified mouse brain. a. PSD-95 positive signal in a 3D rendered image. b. PSD-95 positive signal in a $2 \mathrm{D}$ image. The top is the rostral part, the bottom is the caudal part, the left is the dorsal part, and the right is the ventral part of the mouse brain. c. PSD-95 positive signal in the forebrain. Note: strongly positive signal in the corpus callosum, anterior commissure, fornix; moderate signal in other brain areas. The gray matter of the hippocampus does not have PSD-95 positive signal

has been reported in the in situ hybridization $[4,8,9]$ and immunohistochemistry studies $[5,6]$.

Ventral to the cerebral cortex, which had weak PSD-95 signal, moderate signal was observed in the corpus callosum except the area dorsal to the hippocampus (Fig. $1 \mathrm{~b}$ and c). The positive signal in this stripe was aligned in the same direction as that observed in the caudate putamen, radiating towards the meeting point of the stria terminalis and the internal capsule, which is consistent with results from other studies $[4-6,9]$. The hippocampal fissure showed a narrow line of weak signal (Fig. $1 \mathrm{~b}$ and c), which is in contrast to what was reported in a study using lacZ [6]. In a human postmortem study, it was reported that schizophrenia and bipolar patients showed a lower level of PSD-95 in the hippocampus, especially in the molecular layer of the dentate gyrus, suggesting the diagnostic value of PSD-95 in the reported diseases [10].

Strong signal was also found in the anterior part of the anterior commissure, the fornix, the optic nerve, and the cerebral peduncle ventral to the substantia nigra (Fig. 1b and c). This is in a clear contrast to what has been reported in the lacZ gene knockin mice [6] and other in situ hybridization $[4,9]$ as well as immunohistochemical 
studies [5]. In those studies, most if not all fiber tracts are void of PSD-95 signal. Moderate signal was observed in the shell of accumbens nucleus, the ventral pallidum, and the nucleus of horizontal diagonal band, the lateral preoptic area, and the majority of hypothalamic nuclei. In the thalamus, its rostral half had moderate PSD-95 signal, whereas the caudal half did not have or had weak PSD-95 signal (Fig. 1b and c). This is different from what has been reported by Porter et al. [6].

Positive signal was observed in the majority of midbrain nuclei and nuclei in the rostral hindbrain (the rest of the hindbrain and cerebellum were cut off). Positive signal in the midbrain and the hypothalamus tended to follow the course of large fibers travelling rostrocaudally. In the middle part of the midbrain in sagittal sections, a small band of signal appeared to travel dorsoventrally. Ventral to this band, positive signals converge towards the ventral surface of the hindbrain (Fig. 1b). These features were not observed in previous in situ hybridization and immunohistochemical studies.

The present study showed the expression of PSD-95 in the mouse brain using an emerging $3 \mathrm{D}$ technique advanced CUBIC and this study confirmed some previous reports about PSD-95 in the majority of brain nuclei (Additional file 1). We showed that some fiber bundles were positive for PSD95, which has not been reported in previous studies. To verify this concern, we used Western blot (not shown) and confirmed the specificity of our PSD95 antibody. Our finding might be true due to the fact that lipid of the neuropil has been removed by the clearing solution and the antibody can easily bind to the epitope of PSD-95, whereas in conventional sections, there is no such a step and the interaction between PSD-95 and its antibody may be blocked to some extent, especially in a fiber bundle where the fibers are tightly bound to each other.

The present study did not include the entire hindbrain due to difficulty in mounting the clarified tissue, which was very soft, to the glass capillary for imaging. Based on the findings from the rostral hindbrain, which are similar to what has been shown in in situ hybridization studies [4, 9], it is expected that the majority of hindbrain nuclei will have weak to moderate PSD-95 signal. The cerebellum might have a higher level of signal than the other areas in the hindbrain as indicated by the in situ hybridization studies.

The advanced CUBIC is an efficient technique in clarifying the mouse brain tissue (Additional file 2). For the same reason, it might lead to more protein loss than passive Clear Lipid-exchanged Acrylamide-hybridized Rigid Imaging/ Immunostaining/In situ hybridizationcompatible Tissue hYdrogel (CLARITY) and other similar techniques. Currently, no method is ideal for preserving protein and rendering tissue transparent for imaging. Better clearing and imaging techniques with longer working distance will be in demand in order to show tissue integrity in the $3 \mathrm{D}$ video.

\section{Additional files}

Additional file 1: 3D video of fluorescent PSD-95 signal reconstructed using Arivis. Strong fluorescent signal was observed in the large fiber bundles such as the anterior commissure, fornix, stria terminalis of the thalamus, and corpus callosum. Weak to moderate signal was observed in a large number of nuclei in the forebrain and midbrain. (AVI $34006 \mathrm{~kb}$ )

Additional file 2: Materials and methods. (DOC 30 kb)

\section{Abbreviations}

2D: 2 dimensional; 3D: 3 dimensions; 4D: 4 dimensional; ac: Anterior commissure; acp: Anterior commissure, posterior part; AV: Anteroventral thalamic nucleus; cc: Corpus callosum; CLARITY: Clear Lipid-exchanged Acrylamide-hybridized Rigid Imaging/ Immunostaining/In situ hybridizationcompatible Tissue hYdrogel; CPu: Caudate putamen; CUBIC: Clear, Unobstructed Brain/Body Imaging Cocktails and Computational analysis; DG: Dentate gyrus; DISC1-LI: Disrupted in schizophrenia 1-locus impairment; DLG4: Disc large homolog 4 gene; f: Fornix; fi: Fimbria of hippocampus; ic: Internal capsule; MAGUK: Membrane associated guanylate kinase; MDL: Lateral part of the mediodorsal thalamic nucleus; NA: Numeral aperture; OB: Olfactory bulb; opt: Optic tract; PBS: Phosphate-buffered saline; PC1: Physical containment level 1; PDZ: Post synaptic density protein (PSD95), drosophila disc large tumor suppressor, and zonula occludens-1 protein; PeFLH: Perifornical lateral hypothalamus; PFA: Paraformaldehyde; Po: Posterior thalamic nuclear group; PSD95: Postsynaptic density - 95 kDa protein; S1: Primary sensory cortex; SC: Superior colliculus; sm: Stria medullaris of the thalamus; VLPO: Ventrolateral preoptic nucleus; ZI: Zona incerta

\section{Acknowledgements}

We thank Dr. Michael Carnell for his technical support in light sheet microscope imaging.

\section{Availability of data and materials}

Data including the original stack of images will be available upon request from the corresponding author because the size of the raw data is over 2 TB and can not be uploaded to any available depository

\section{Authors' contributions}

$\mathrm{HL}, \mathrm{XH}, \mathrm{SW}$, and GP conceived the study. HW perfused the mice and dissected the mouse brains. HL performed CUBIC clearing, immunofluorescent staining, and drafting the manuscript. RF performed light sheet microscope imaging. All authors read and approved the final manuscript.

\section{Ethics and consent to participate}

The animal experiment was approved by the Animal Care and Ethics Committee of The University of New South Wales (approval number: 14/94A) and conducted in compliance with the revised Animals (Scientific Procedures) Act 1986 in the UK.

\section{Consent to publish}

Not applicable.

\section{Competing interests}

All authors declare that there are no competing interests.

\section{Publisher's Note}

Springer Nature remains neutral with regard to jurisdictional claims in published maps and institutional affiliations.

\section{Author details}

${ }^{1}$ Neurology Department, Shanghai No.1 People's Hospital (in the process of affiliation), School of Medicine, Tongji University, Shanghai 200081, China.

${ }^{2}$ Neuroscience Research Australia, Randwick, NSW 2031, Australia. ${ }^{3}$ School of 
Medicine, Western Sydney University, Campbelltown, NSW 2560, Australia ${ }^{4}$ Illawarra Health and Medical Research Institute, Wollongong, NSW 2522, Australia. ${ }^{5}$ Biomedical Imaging Facility, Mark Wainwright Analytical Centre, The University of New South Wales, Kensington, NSW 2052, Australia.

\section{Received: 8 August 2018 Accepted: 5 September 2018}

\section{Published online: 12 September 2018}

\section{References}

1. Baucum AJ 2nd. Proteomic analysis of postsynaptic protein complexes underlying neuronal plasticity. ACS Chem Neurosci. 2017:8(4):689-701.

2. Won S, Incontro S, Nicoll RA, Roche KW. PSD-95 stabilizes NMDA receptors by inducing the degradation of STEP61. Proc Natl Acad Sci U S A. 2016;113:E4736-44.

3. Malenka RC, Bear MF. LTP and LTD: an embarrassment of riches. Neuron. 2004:44:5-21.

4. Fukaya M, Ueda H, Yamauchi K, Inoue $\mathrm{Y}$, Watanabe M. Distinct spatiotemporal expression of mRNAs for the PSD-95 / SAP90 protein family in the mouse brain. Neurosci Res. 1999:33:111-8.

5. Fukaya $\mathrm{M}$, Watanabe M. Improved immunohistochemical detection of postsynaptically located PSD-95 / SAP90 protein family by protease section pretreatment: a study in the adult mouse brain. J Comp Neurol. 2000;426:572-86.

6. Porter K, Komiyama NH, Vitalis T, Kind PC, Grant SG. Differential expression of two NMDA receptor interacting proteins, PSD-95 and SynGAP during mouse development. Eur J Neurosci. 2005:21(2):351-62.

7. Susaki EA, Tainaka K, Perrin D, Yukinaga H, Kuno A, Ueda HR. Advanced CUBIC protocols for whole-brain and whole-body clearing and imaging. Nat Protoc. 2015;10(11):1709-27.

8. Shu F, Ohno K, Wang T, Kuriyama K, Ueki T, Kanayama N, Sato K. Developmental changes in PSD-95 and Narp mRNAs in the rat olfactory bulb. Brain Res Dev Brain Res. 2001;132(1):91-5.

9. Yao WD, Gainetdinov RR, Arbuckle MI, Sotnikova TD, Cyr M, Beaulieu JM, Torres GE, et al. Identification of PSD-95 as a regulator of dopaminemediated synaptic and behavioral plasticity. Neuron. 2004;41(4):625-38.

10. Toro C, Deakin JF. NMDA receptor subunit NRI and postsynaptic protein PSD-95 in hippocampus and orbitofrontal cortex in schizophrenia and mood disorder. Schizophr Res. 2005;80(2-3):323-30.

Ready to submit your research? Choose BMC and benefit from:

- fast, convenient online submission

- thorough peer review by experienced researchers in your field

- rapid publication on acceptance

- support for research data, including large and complex data types

- gold Open Access which fosters wider collaboration and increased citations

- maximum visibility for your research: over $100 \mathrm{M}$ website views per year

At $\mathrm{BMC}$, research is always in progress.

Learn more biomedcentral.com/submissions 